University of Nebraska - Lincoln

DigitalCommons@University of Nebraska - Lincoln

July 2004

\title{
Training of the exchange-bias effect: A simple analytic approach
}

Christian Binek

University of Nebraska-Lincoln, cbinek@unl.edu

Follow this and additional works at: https://digitalcommons.unl.edu/physicsbinek

Part of the Physics Commons

Binek, Christian, "Training of the exchange-bias effect: A simple analytic approach" (2004). Christian Binek Publications. 23.

https://digitalcommons.unl.edu/physicsbinek/23

This Article is brought to you for free and open access by the Research Papers in Physics and Astronomy at DigitalCommons@University of Nebraska - Lincoln. It has been accepted for inclusion in Christian Binek Publications by an authorized administrator of DigitalCommons@University of Nebraska - Lincoln. 


\title{
Training of the exchange-bias effect: A simple analytic approach
}

\author{
Christian Binek* \\ Department of Physics and Astronomy and the Center for Materials Research and Analysis, Ferguson Hall, University of Nebraska, \\ Lincoln, Nebraska 68588-0111, USA
}

(Received 2 December 2003; published 20 July 2004)

\begin{abstract}
Training of the exchange bias effect in antiferro-/ferromagnetic heterostructures is considered in the theoretical framework of spin configurational relaxation, which is activated through consecutively cycled hysteresis loops. The corresponding exchange bias fields, $\mu_{0} H_{\mathrm{EB}}(n)$, reveal relaxation from the initial state, $n=1$, of high antiferromagnetic interface magnetization to the equilibrium state of reduced magnetization in the limit of large $n$. The evolution of $\mu_{0} H_{\mathrm{EB}}$ vs $n$ is calculated with the help of a discretized Landau-Khalatnikov equation, where the continuous time parameter is replaced by the loop index $n$. The result reveals the origin of the well established but hitherto unexplained power-law decay of $\mu_{0} H_{\mathrm{EB}}(n)$ for $n>1$. Moreover, in contrast with the breakdown of the power-law behavior at $n=1$, the relaxation approach describes the training effect for $n \geqslant 1$. The full capability of the theory is explored in comparison with experimental results obtained recently on a $\mathrm{NiO}(001) / \mathrm{Fe}(110)$ heterostructure.
\end{abstract}

DOI: $10.1103 /$ PhysRevB.70.014421

PACS number(s): 75.60.-d, 75.70.Cn

Unidirectional magnetic anisotropy in heterostructures of exchange coupled antiferromagnetic (AF) and ferromagnetic (FM) thin films is induced after field cooling the system to below the Néel temperature of the AF pinning layer. It gives rise to a shift of the FM hysteresis loop along the magnetic field axis by the amount $\mu_{0} H_{\mathrm{EB}}$. This exchange bias (EB) effect turns out to decrease monotonically when cycling the heterostructure through consecutive hysteresis loops. The resulting $\mu_{0} H_{\mathrm{EB}}$ vs $n$-dependence displays the so called training effect. Here $n$ labels the number of loops, which have been cycled after preparation of the initial state via the fieldcooling procedure described above. The strength of the training effect depends significantly on the properties of the AF pinning layer of the heterostructure. Pronounced training effects have been found in heterosystems involving polycrystalline AF pinning layers ${ }^{1-3}$ while in single crystalline pinning systems the effect is expected to be small. ${ }^{4}$

Basically, a nonstationary EB effect indicates that the spin structure of the AF/FM heterostructure deviates from its equilibrium configuration. The gradual decrease of $\mu_{0} H_{\mathrm{EB}}$ with increasing loop index $n$ is a macroscopic fingerprint of configurational rearrangements of the spin structure towards equilibrium. The intimate connection between the EB effect and nonequilibrium AF spin structures at the AF/FM interface is certainly one major reason for the complexity of the EB phenomenon. It is the primary task of microscopic EB theories to explain the origin of the AF interface magnetization and its evolution with temperature, magnetic field, loop index and other external parameters. Hence, it is not surprising, that, up to now, there are a number of models, which try to elucidate the microscopic origin of the exchange bias effect. $^{5-7}$ Although, it remains doubtful whether a unique mechanism exists, which describes all aspects of the variety of experimental findings, ${ }^{4,8}$ it became common sense that a net interface magnetization of the antiferromagnet is necessary in order to observe the exchange bias effect.

The Meiklejohn Bean expression $\mu_{0} H_{\mathrm{EB}}=-J\left(S_{\mathrm{AF}} S_{\mathrm{FM}}\right) /$ $\left(t_{\mathrm{FM}} M_{\mathrm{FM}}\right)$ describes the dependence of the bias field on a phenomenological coupling $J$ between the FM and AF interface magnetization $S_{\mathrm{FM}}$ and $S_{\mathrm{AF}}$, respectively, while $t_{\mathrm{FM}}$ and $M_{\mathrm{FM}}$ are the thickness and the saturation magnetization of the FM layer. ${ }^{9,10}$ Although this phenomenological approach does not tackle the microscopic origin of the AF interface magnetization, it suggests that the training effect originates from the training of $S_{\mathrm{AF}}$. Microscopically, the $n$-dependence of $S_{\mathrm{AF}}$ reflects the reorientation of $\mathrm{AF}$ domains at the AF/FM interface. AF domain states have been extensively studied, for instance, in the case of NiO. ${ }^{11-17}$

In fact, it has been recently shown, that the total magnetic moment of a $\mathrm{NiO}(001) / \mathrm{Fe}(110)$ heterostructure is reduced with consecutively cycled hysteresis loops. ${ }^{18,19}$ Since the FM top layer of the AF/FM heterostructure is saturated after each loop, it is obvious, that the successive reduction of the magnetic moment takes place in the AF pinning component. The AF spin reorientation is initiated at the AF/FM interface. Here, the coupling between $S_{\mathrm{AF}}$ and $S_{\mathrm{FM}}$ triggers the relaxation process during the magnetization reversal of the FM layer. It is therefore reasonable to expect, that $S_{\mathrm{AF}}$ contributes to the reduction of the magnetic moment of the AF pinning layer. The same conclusion on the microscopic origin of the training effect has been drawn recently for $\mathrm{Co} / \mathrm{CoO} / \mathrm{Co}_{1-x} \mathrm{Mg}_{x} \mathrm{O}$ heterostructures ${ }^{20}$ Here, the initial AF domain state can be controlled by random fields, which act on the AF order parameter. Their strength depends on the concentration $x$ of the diamagnetic dilution and the freezing field, respectively. ${ }^{21,22}$ Corresponding Monte Carlo simulations reveal irreversible reorientation processes of the $\mathrm{AF}$ spin structure, which give rise to decreasing AF interface magnetization upon subsequent magnetization reversal of the FM layer. ${ }^{23}$

In the framework of this microscopic picture it is reasonable to map the training effect of the EB onto a training effect of $S_{\mathrm{AF}}$ by $\mu_{0} H_{\mathrm{EB}}(n)=K S_{\mathrm{AF}}(n)$, where the proportionality constant $K$ is independent of the loop index $n$ and phenomenologically given by $K=-J S_{\mathrm{FM}} /\left(M_{\mathrm{FM}} t_{\mathrm{FM}}\right)$ in accordance with the Meiklejohn Bean expression. 
It is the aim of the following analysis to derive $S_{\mathrm{AF}}(n)$ in the framework of a simple analytical approach and map it directly onto the training effect of the EB field. In particular, it is intended to deduce the surprisingly simple and experimentally often observed power-law

$$
\mu_{0} H_{\mathrm{EB}}(n)-\mu_{0} H_{\mathrm{EB}}^{e}=\frac{\kappa}{\sqrt{n}},
$$

where $\kappa$ is a system dependent constant and $\mu_{0} H_{\mathrm{EB}}^{e}$ is the exchange bias field in the limit of infinite loops. ${ }^{24}$ The simplicity of this expression on the one hand and its applicability in the case of significantly distinct systems is asking for a simple physical basis underlying Eq. (1), which we are going to reveal.

As a starting point we define the equilibrium AF interface magnetization $S_{\mathrm{AF}}^{e}=\lim _{n \rightarrow \infty} S_{\mathrm{AF}}(n)$, which has to exist in the case of a strictly monotonic decrease of $\left|S_{\mathrm{AF}}(n)\right|$. Each positive or negative deviation $\delta S_{n}=S_{\mathrm{AF}}(n)-S_{\mathrm{AF}}^{e}$ of the AF interface magnetization from its equilibrium value will increase the total free energy $F$ of the system by $\Delta F$. If we assume $\Delta F(\delta S)=\Delta F(-\delta S)$, a series expansion of $\Delta F$ up to fourth order in $\delta S_{n}$ reads

$$
\Delta F=\frac{1}{2} a \delta S_{n}^{2}+\frac{1}{4} b \delta S_{n}^{4}+O\left(\delta S_{n}^{6}\right),
$$

where powers of the order $O\left(\delta S_{n}^{6}\right)$ are negligible if $\left|\delta S_{n}\right|$ is sufficiently small. Note, that it is not intended to describe a phase transition by the expansion (2) although there is a close formal analogy. In particular, it is obsolete to consider an expansion up to $O\left(\delta S_{n}^{6}\right)$ from the point of view of thermodynamic stability. The latter argument is known from the Landau theory of first order phase transitions where $b<0$ requires higher order stabilization.

The relaxation of the system towards equilibrium is determined by the Landau-Khalatnikov (LK) equation ${ }^{25}$

$$
\xi \dot{S}_{\mathrm{AF}}=-\frac{\partial \Delta F}{\partial S_{\mathrm{AF}}},
$$

where $\xi$ is a phenomenological damping constant and $\dot{S}_{\mathrm{AF}}$ is the derivative of $S_{\mathrm{AF}}$ with respect to time. Equation (3) has a broad range of applications when $S_{\mathrm{AF}}$ is considered as the order parameter of a system. The LK-equation has been derived, e.g., from Lagrangian formalism. Here, the potential is given by $F$ and strong dissipation has to be taken into account, which gives rise to over-critical damping in the resulting equation of motion. ${ }^{25}$ The latter condition is fulfilled in the case of the training effect, since the evolution of the AF interface magnetization is expected to be very slow in comparison with the microscopic spin fluctuations. On the timescale of the EB experiments the temporal change of $S_{\mathrm{AF}}$ is even negligible unless the system is triggered by the magnetization reversal of the FM top layer. Hence, in order to tackle the training effect in the framework of the LK-approach, the left hand side of Eq. (3) has to be discretized.

Figure 1 shows a sketch of the temporal evolution of the AF interface magnetization. While $S_{\mathrm{AF}}$ is apparently constant in the time interval $\Delta t$ between two subsequent hysteresis

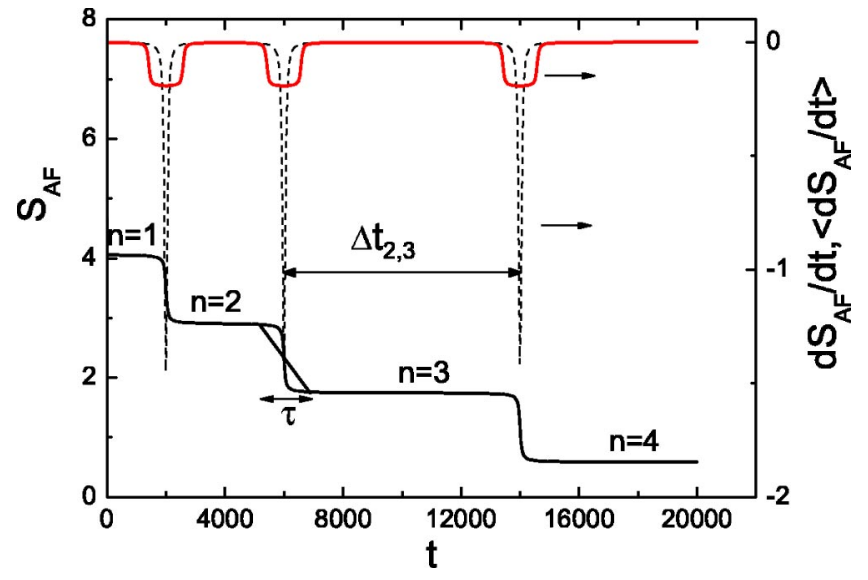

FIG. 1. Sketch of the temporal evolution of $S_{\mathrm{AF}}$. Measurement of a single hysteresis loop requires the typical time interval $\tau$. The slope of the straight line visualizes the average value of the derivative $\left\langle d S_{\mathrm{AF}} / d t\right\rangle_{\tau}$ during the 2 nd loop. Dashed and solid lines (right axis) show the derivative $d S_{\mathrm{AF}} / d t$ vs $t$ and the corresponding average $\left\langle d S_{\mathrm{AF}} / d t\right\rangle_{\tau}$ vs $t$, respectively. $n=1,2,3$, and 4 label the regions of apparently constant $S_{\mathrm{AF}}$. The crossover from the $n$th to the $(n$ +1 )th plateau is the temporal regime where relaxation of $S_{\mathrm{AF}}$ is triggered by the $n$th hysteresis loop. $\Delta t_{2,3}$ labels the time interval between the 2 nd and 3 rd hysteresis loop.

loops, the $n$th FM hysteresis stimulates relaxation of $S_{\mathrm{AF}}$ from $S_{\mathrm{AF}}(n)$ to $S_{\mathrm{AF}}(n+1)$. The transition takes place during the time interval $\tau$, which is required for the measurement of the loop. The change of $S_{\mathrm{AF}}$ is given by the average value of the derivative $d S_{\mathrm{AF}} / d t$ during the $n$th hysteresis loop. It reads

$$
\frac{S_{\mathrm{AF}}(n+1)-S_{\mathrm{AF}}(n)}{\tau}=\left\langle\frac{d S_{\mathrm{AF}}}{d t}\right\rangle_{\tau},
$$

with

$$
\left\langle\frac{d S_{\mathrm{AF}}}{d t}\right\rangle_{\tau}=\frac{1}{\tau} \int_{-\tau / 2}^{\tau / 2} d t \frac{d S_{\mathrm{AF}}}{d t}
$$

(see right axis of Fig. 1, solid and dashed lines are sketches of $\left\langle d S_{\mathrm{AF}} / d t\right\rangle_{\tau}$ and $d S_{\mathrm{AF}} / d t$, respectively).

Replacement of $\dot{S}_{\mathrm{AF}}$ by $\left\langle d S_{\mathrm{AF}} / d t\right\rangle_{\tau}$ converts the differential equation (3) into a difference equation. The resulting implicit sequence reads

$$
\tilde{\xi}\left(S_{\mathrm{AF}}(n+1)-S_{\mathrm{AF}}(n)\right)=-\delta S_{n}\left(a+b \delta S_{n}^{2}\right),
$$

where $\tilde{\xi}=\xi / \tau$ and the right hand side is given by the derivative of Eq. (2). In the case of a strictly monotonic decrease of $\left|S_{\mathrm{AF}}(n)\right|, a>0$ causes necessarily an asymptotic decay of the type $S_{\mathrm{AF}}(n) \propto e^{-a n}+S_{\mathrm{AF}}^{e}$, when finally $\delta S_{n}^{3} \ll \delta S_{n}$. Exponential relaxation, which is generically faster than any potential decay, is typically observed, when spin correlation becomes negligible. In the case of exchange bias, however, large $\mathrm{AF}$ spin correlation is essential in order to pin the FM layer during its magnetization reversal. Exchange bias and the training effect disappear necessarily above the blocking temperature where AF spin correlation significantly levels off. Hence, nonexponential relaxation has to be expected below 
the blocking temperature. It requires $a=0$ and simplifies Eq. (6) according to

$$
S_{\mathrm{AF}}(n+1)-S_{\mathrm{AF}}(n)=-\frac{b}{\widetilde{\xi}} \delta S_{n}^{3} .
$$

Note the close analogy of expression (7) with the phenomenon of critical slowing down. ${ }^{26}$ In the theory of phase transitions a dynamic differential equation of the Bernoulli type can be derived in the molecular field approximation for interacting Ising spins from the Glauber model. ${ }^{27} \mathrm{Here}$, at the critical temperature, the driving force into equilibrium scales with the third power of the order parameter, while relaxation is the response of the spin system on a steplike change of the applied magnetic field. One may speculate, that the formal close analogy between critical slowing down and the training effect originates from the physics of large spin fluctuations, which play an important role in the case of both phenomena.

Multiplication of Eq. (7) with the proportionality constant $K$ and substitution of $\delta S_{n}=S_{\mathrm{AF}}(n)-S_{\mathrm{AF}}^{e}$ maps the $n$-dependence of $S_{\mathrm{AF}}$ onto the EB field. It reads

$$
\mu_{0}\left(H_{\mathrm{EB}}(n+1)-H_{\mathrm{EB}}(n)\right)=-\gamma\left(\mu_{0}\left(H_{\mathrm{EB}}(n)-H_{\mathrm{EB}}^{e}\right)\right)^{3},
$$

with

$$
\gamma=b /\left(K^{2} \widetilde{\xi}\right) .
$$

To relate the empirical parameter $\kappa$ in Eq. (1) to the physical parameter $\gamma$ in Eq. (8) in the limit $n \gg 1$, we substitute Eq. (1) into (8) and expand the $(n+1)$-dependent term according to $1 / \sqrt{n+1} \simeq 1 / \sqrt{n}-1 /(2 n \sqrt{n})$. Note, that already at $n=3$ the approximation holds within an error of less than 5\%. After some rearrangements one obtains the relation

$$
\kappa=K \sqrt{\frac{\tilde{\xi}}{2 b}},
$$

which allows for physical interpretation. Let us, for instance, consider a steep potential $F$. It is characterized by a large expansion coefficient $b$ in accordance with Eq. (2). In this case, deviations from the equilibrium state are energetically highly unfavorable. Hence, $\delta S_{n}$ is expected to be small and consequently the training of the EB effect will be small. This is empirically expressed in terms of a small value of $\kappa$ in accordance with Eq. (10). In addition, the training effect requires coupling between the $\mathrm{AF}$ and $\mathrm{FM}$ layer which triggers the rearrangement of the spin configuration in the antiferromagnet during the magnetization reversal. The phenomenological coupling constant $J$ is proportional to $K$ and, hence, Eq. (10) expresses the increase of $\kappa$ with increasing coupling strength. Finally, the damping constant $\tilde{\xi}$ can be considered as a typical inverse relaxation time or, in the framework of the discretized equation, as a relaxation rate. The latter scales with the inverse number of cycles, which are required in order to approach equilibrium. A large $\widetilde{\xi}$ gives rise to significant relaxation already after a few cycles. Hence, $\kappa$ increases with increasing $\tilde{\xi}$ in accordance with Eq. (10).

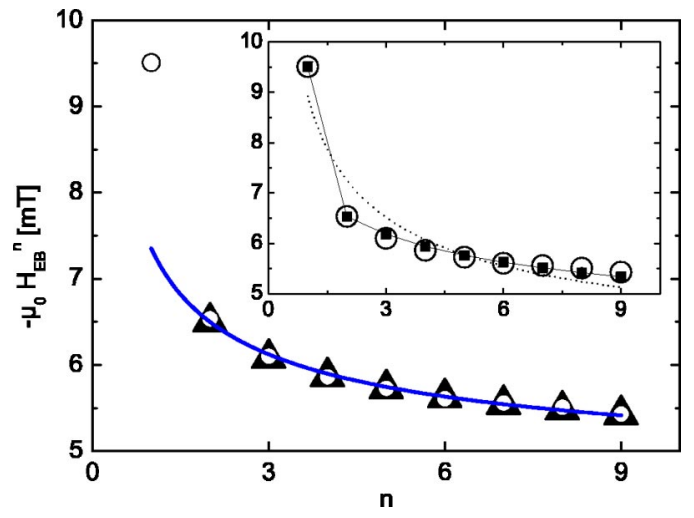

FIG. 2. Training effect of the exchange bias $\mu_{0} H_{\mathrm{EB}}$ vs $n$ (open circles) of a $\mathrm{NiO}(001) / \mathrm{Fe}(110)$ heterostructure (Ref. 18). Solid line shows the best fit of Eq. (1) to the data for $n>1$. The result of the fit is extrapolated down to $n=1$ in order to indicate the breakdown of the power-law behavior at $n=1$. Triangles show the data generated from the recursive sequence (8) with $\mu_{0} H_{\mathrm{EB}}^{e}=4.45 \mathrm{mT}$ (result adopted from the power-law fit) and $\gamma=0.049(\mathrm{mT})^{-2}$ [optimized in accordance with Eq. (11)]. Inset shows again the experimental data (open circles) as reference, while the squares display the result of the best fit (for nonlinear fitting procedure see text) of sequence (8) to the entire data set for $n \geqslant 1$. Straight lines connecting the squares are a guide to the eyes only. The dashed line is a best fit of Eq. (1) to the entire experimental data.

Figure 2 shows the experimental data of $\mu_{0} H_{\mathrm{EB}}$ vs $n$ (open circles) obtained recently from measurements of the magnetic hysteresis loops on a $\mathrm{NiO}(001) / \mathrm{Fe}(110)$ heterostructure. ${ }^{18}$ The measurements have been carried out with the help of a superconducting quantum interference device magnetometer at $T=5 \mathrm{~K}$ in planar field geometry. For experimental details see Ref. 18. The solid line in Fig. 2 shows the best fit of the empirical Eq. (1) for data with loop index $n>1$. The resulting fitting parameters read $\mu_{0} H_{\mathrm{EB}}^{e}$ $=4.45 \mathrm{mT}$ and $\kappa=2.90 \mathrm{mT}$. The fit shows perfect agreement with the data for $n>1$. It is obvious, that a fit of the data will also work if an additional free parameter is involved and the restriction to nonexponential asymptotic relaxation is given up. In this case, however, one has to conclude, that the experimental data are satisfactorily described for $a=0$, which asymptotically corresponds to Eq. (1) as outlined above. This means, that the phenomenological approach contains the empirical $1 / \sqrt{n}$-dependence.

In accordance with previous experience, however, its extrapolation down to $n=1$ reveals the breakdown of the power-law behavior. Alternatively, $\mu_{0} H_{\mathrm{EB}}$ vs $n$ is calculated from the recursive sequence (8) for $n>1$. The solid triangles show the result. Here the sequence has been started with the experimental data point $\mu_{0} H_{\mathrm{EB}}(n=2)$. The optimized parameter $\gamma=0.049(\mathrm{mT})^{-2}$ is calculated from the experimental data according to

$$
\gamma=\frac{1}{(N-1)} \sum_{n=2}^{N} \frac{\mu_{0}\left(H_{\mathrm{EB}}(n)-H_{\mathrm{EB}}(n+1)\right)}{\left(\mu_{0}\left(H_{\mathrm{EB}}(n)-H_{\mathrm{EB}}^{e}\right)\right)^{3}},
$$

where $\mu_{0} H_{\mathrm{EB}}^{e}=4.45 \mathrm{mT}$ has been used as additional input from the power-law fit. There is apparent perfect coincidence 
with the data for $n>1$ and, hence, with the power-law. However, comparison of $\gamma=0.049(\mathrm{mT})^{-2}$ [optimized according to Eq. (11)] with $\gamma=1 /\left(2 \kappa^{2}\right)=0.059(\mathrm{mT})^{-2}$ reveals small differences between both approaches. The latter $\gamma$-value has to be expected in the case of nonconstraining validity of the power-law in accordance with Eqs. (10) and (9). In addition, it is remarkable, that a backward extrapolation of the sequence (8) to $\mu_{0} H_{\mathrm{EB}}(n=1)$ becomes impossible, since there is no positive real solution for the given parameters $\gamma$ and $\mu_{0} H_{\mathrm{EB}}^{e}$. This indicates the generic difference between the power-law and the recursive sequence at $n=1$.

The extraordinary strong decay of the EB field between the first and the second loop is obviously not described by the empirical power-law Eq. (1) (see Fig. 2). The outstanding role of $\mu_{0} H_{\mathrm{EB}}(n=1)$ stimulated recent experimental and theoretical work. ${ }^{28-30}$ Radu et al. found that the down and up branches of the hysteresis loops of a $\mathrm{CoO} / \mathrm{Co}$ bilayer follow different mechanisms of magnetization reversal. While coherent rotation has been observed at the coercive field $\mathrm{H}_{c 2}$, domain nucleation and wall propagation dominate in the vicinity of $H_{c 1}$. The large training effect at $n=1$ originates here from pure $180^{\circ}$ domain wall movement, which takes place at $H_{c 1}$ during the first magnetization reversal. Very recent Monte Carlo simulations show that the asymmetry of the reversal modes depends on the angle between the AF easy axis and the applied magnetic field in heterostructures which fulfill the prerequisites of the domain state model. ${ }^{31}$

The phenomenological relaxation approach presented here does not depend on these microscopic details. It describes, merely, in which way deviations from the equilibrium of the AF interface magnetization affect the free energy. The expansion of the free energy in accordance with Eq. (2) is based on the assumption of time inversion symmetry. It is experimentally well known, that the absolute values of the EB field remain unchanged when the preparation of the initial states takes place in the presence of inverted internal freezing fields. ${ }^{32,33}$ Hence, time inversion symmetry of $F$ is expected to be fulfilled. Therefore, it is reasonable to assume that the sequence (8) holds for $n \geqslant 1$. In order to check its full capability we look for optimized parameters $\gamma$ and $\mu_{0} H_{\mathrm{EB}}^{e}$ which are independent of the results of the power-law fit. Therefore,

$$
f=\sum_{n}\left(H_{\mathrm{EB}}(n)-\gamma \mu_{0}^{2}\left(H_{\mathrm{EB}}(n)-H_{\mathrm{EB}}^{e}\right)^{3}-H_{\mathrm{EB}}(n+1)\right)^{2},
$$

is minimized with respect to $\gamma$ and $\mu_{0} H_{\mathrm{EB}}^{e}$. The conditions $\partial f / \partial \gamma=0$ and $\partial f / \partial\left(\mu_{0} H_{\mathrm{EB}}^{e}\right)=0$ yield the two equations

$$
\gamma_{j}=\frac{\sum_{n}\left(H_{\mathrm{EB}}(n+1)-H_{\mathrm{EB}}(n)\right)\left(H_{\mathrm{EB}}(n)-H_{\mathrm{EB}}^{e}\right)^{1+j}}{\mu_{0}^{2} \sum_{n}\left(H_{\mathrm{EB}}(n)-H_{\mathrm{EB}}^{e}\right)^{4+j}},
$$

for $j=1$ and 2, respectively. There is only one real solution of $\gamma_{1}\left(\mu_{0} H_{\mathrm{EB}}^{e}\right)=\gamma_{2}\left(\mu_{0} H_{\mathrm{EB}}^{e}\right)$ which yields $\mu_{0} H_{\mathrm{EB}}^{e}=3.66 \mathrm{mT}$ and $\gamma=0.0149(\mathrm{mT})^{-2}$. The inset of Fig. 2 displays the result of the fit (squares) and the very satisfactory coincidence with the entire experimental data set (circles). Note, that the number of 2 free fitting parameters has not been increased in comparison with Eq. (1). However, both parameters deviate significantly from the results obtained by the power-law fit for $n>1$. It is remarkable, however, that the power-law fit for $n \geqslant 1$ (see dotted line in the inset of Fig. 2) yields $\mu_{0} H_{\mathrm{EB}}^{e}=3.23 \mathrm{mT}$ and $\kappa=5.69 \mathrm{mT}$. The latter value corresponds to $\gamma=1 /\left(2 \kappa^{2}\right)=0.0154(\mathrm{mT})^{-2}$. Although the quality of the power-law fit is poor, the resulting parameters come surprisingly close to those obtained from the best fit of the recursive sequence.

In summary, the training effect in EB heterostructures is considered in the framework of nonequilibrium thermodynamics. Consecutively cycled hysteresis loops of the FM top layer trigger the spin configurational relaxation of the $\mathrm{AF}$ interface magnetization towards equilibrium. The waiting time between subsequent loops is irrelevant for the relaxation process. This gives rise to an interesting discrete dynamic behavior, which is described here in the framework of a discretized Landau-Khalatnikov equation. The phenomenological energy landscape, which controls the relaxation process, depends exclusively on the deviation of the AF interface magnetization from its equilibrium value. In particular, it is independent from microscopic details of the magnetization reversal of the FM top layer. The presented approach provides the phenomenological origin of the hitherto unexplained power-law decay of the EB field with increasing loop index $n>1$. Moreover, the general recursive sequence describes the entire dependence of the EB fields of a $\mathrm{NiO}(001) / \mathrm{Fe}(110)$ heterostructure on the loop index $n$.

I gratefully acknowledge fruitful discussions with Vladimir Fridkin.
*Electronic address: cbinek2@unl.edu

${ }^{1}$ C. Schlenker, S. S. P. Parkin, J. C. Scott, and K. Howard, J. Magn. Magn. Mater. 54, 801 (1986).

${ }^{2}$ K. Zhang, T. Zhao, and M. Fujiwara, J. Appl. Phys. 89, 6910 (2001).

${ }^{3}$ S. G. te Velthuis, A. Berger, and G. P. Felcher, J. Appl. Phys. 87,
5046 (2001).

${ }^{4}$ J. Nogués and I. K. Schuller, J. Magn. Magn. Mater. 192, 203 (1999).

${ }^{5}$ A. P. Malozemoff, Phys. Rev. B 35, 3679 (1987).

${ }^{6}$ R. L. Stamps, J. Phys. D 33, R247 (2000).

${ }^{7}$ P. Miltényi, M. Gierlings, J. Keller, B. Beschoten, G. Güntherodt, 
U. Nowak, and K. D. Usadel, Phys. Rev. Lett. 84, 4224 (2000).

${ }^{8} \mathrm{~A}$ very instructive summary of open questions in the field of EB can be found at http://physics.ucsd.edu/iksgrp/EB/Manifesto.pdf

${ }^{9}$ W. H. Meiklejohn and C. P. Bean, Phys. Rev. 102, 1413 (1956).

${ }^{10}$ W. H. Meiklejohn and C. P. Bean, Phys. Rev. 105, 904 (1956).

${ }^{11}$ W. L. Roth, J. Appl. Phys. 31, 2000 (1960).

${ }^{12}$ W. Kleemann, F. J. Schäfer, and D. S. Tannhauser, J. Magn. Magn. Mater. 15, 415 (1980).

${ }^{13}$ H. Ohldag, A. Scholl, F. Nolting, S. Anders, F. U. Hillebrecht, and J. Stöhr, Phys. Rev. Lett. 86, 2878 (2001).

${ }^{14}$ F. U. Hillebrecht, H. Ohldag, N. B. Weber, C. Bethke, U. Mick, M. Weiss, and J. Bahrdt, Phys. Rev. Lett. 86, 3419 (2001).

${ }^{15}$ H. Ohldag, T. J. Regan, J. Stöhr, A. Scholl, F. Nolting, J. Lüning, C. Stamm, S. Anders, and R. L. White, Phys. Rev. Lett. 87, 247201 (2001)

${ }^{16}$ H. Matsuyama, C. Haginoya, and K. Koike, Phys. Rev. Lett. 85, $646(2000)$

${ }^{17}$ J. A. Borchers, Y. Ijiri, D. M. Lind, P. G. Ivanov, R. W. Erwin, A. Quasba, S. H. Lee, K. V. O. Donovan, and D. C. Dender, Appl. Phys. Lett. 77, 4187 (2000).

${ }^{18}$ A. Hochstrat, Ch. Binek, and W. Kleemann, Phys. Rev. B 66, 092409 (2002).

${ }^{19}$ Ch. Binek, Ising-type Antiferromagnets: Model Systems in Statistical Physics and in the Magnetism of Exchange Bias, Springer Tracts in Modern Physics, Vol. 196 (Springer, Berlin, 2003).

${ }^{20}$ J. Keller, P. Miltényi, B. Beschoten, G. Güntherodt, U. Nowak, and K. D. Usadel, Phys. Rev. B 66, 014431 (2002).

${ }^{21}$ W. Kleemann, Int. J. Mod. Phys. B 7, 2469 (1993).

${ }^{22}$ D. P. Belanger and P. Young, J. Magn. Magn. Mater. 100, 272 (1991).

${ }^{23}$ U. Nowak, K. D. Usadel, J. Keller, P. Miltényi, B. Beschoten, and G. Güntherodt, Phys. Rev. B 66, 014430 (2002).

${ }^{24}$ D. Paccard, C. Schlenker, O. Massenet, R. Montmory, and A. Yelon, Phys. Status Solidi 16, 301 (1966).

${ }^{25}$ G. Vizdrik, S. Ducharme, V. M. Fridkin, and G. Yudin, Phys. Rev. B 68, 094113 (2003).

${ }^{26}$ H. Haken, Light, Vol. 2 (North-Holland Physics Publishing, 1985), p. $314 \mathrm{ff}$.

${ }^{27} \mathrm{H}$. Eugene Stanley, Introduction to Phase Transitions and Critical Phenomena (Clarendon Press, Oxford, 1971), p. $280 \mathrm{ff}$.

${ }^{28}$ M. R. Fitzsimmons, P. Yashar, C. Leighton, I. K. Schuller, J. Nogués, C. F. Majkrzak, and J. A. Dura, Phys. Rev. Lett. 84, 3986 (2000).

${ }^{29}$ F. Radu, M. Etzkorn, R. Siebrecht, T. Schmitte, K. Westerholt, and H. Zabel, J. Magn. Magn. Mater. 240, 251 (2002).

${ }^{30}$ F. Radu, M. Etzkorn, R. Siebrecht, T. Schmitte, K. Westerholt, and H. Zabel, Phys. Rev. B 67, 134409 (2003).

${ }^{31}$ B. Beckmann, U. Nowak, and K. D. Usadel, Phys. Rev. Lett. 91, 187201 (2003).

${ }^{32}$ P. Miltényi, M. Gierlings, M. Bamming, U. May, G. Güntherodt, J. Nogués, and I. K. Schuller, Appl. Phys. Lett. 75, 2304 (1999).

${ }^{33} \mathrm{Ch}$. Binek, Xi Chen, A. Hochstrat, and W. Kleemann, J. Magn. Magn. Mater. 240, 257 (2002). 